\title{
Article \\ Reusable and pH-Stable Luminescent Sensors for Highly Selective Detection of Phosphate
}

\author{
Do Yeob Kim ${ }^{1,+}{ }^{\oplus}$, Dong Gyu Kim ${ }^{2,+}$, Bongjin Jeong ${ }^{1}\left(\mathbb{D}\right.$, Young Il Kim ${ }^{2}$, Jungseok Heo ${ }^{2, *}$ \\ and Hyung-Kun Lee ${ }^{1, *(D)}$ \\ 1 ICT Creative Research Laboratory, Electronics \& Telecommunications Research Institute, \\ Daejeon 34129, Korea; nanodykim@etri.re.kr (D.Y.K.); jbj0919@etri.re.kr (B.J.) \\ 2 Department of Chemistry, Chungnam National University, Daejeon 34134, Korea; \\ kdg05262@naver.com (D.G.K.); yi4902@naver.com (Y.I.K.) \\ * Correspondence: jungseokheo@cnu.ac.kr (J.H.); hklee@etri.re.kr (H.-K.L.); Tel.: +82-42-860-5857 (H.-K.L.) \\ + These authors contributed equally to this work.
}

check for updates

Citation: Kim, D.Y.; Kim, D.G.; Jeong, B.; Kim, Y.I.; Heo, J.; Lee, H.-K. Reusable and $\mathrm{pH}$-Stable Luminescent Sensors for Highly Selective Detection of Phosphate. Polymers 2022, 14, 190. https://doi.org/ $10.3390 /$ polym 14010190

Academic Editor: Chen-I Yang

Received: 2 December 2021

Accepted: 31 December 2021

Published: 4 January 2022

Publisher's Note: MDPI stays neutral with regard to jurisdictional claims in published maps and institutional affiliations.

Copyright: (C) 2022 by the authors. Licensee MDPI, Basel, Switzerland. This article is an open access article distributed under the terms and conditions of the Creative Commons Attribution (CC BY) license (https:// creativecommons.org/licenses/by/ $4.0 /)$.

\begin{abstract}
Phosphate sensors have been actively studied owing to their importance in water environment monitoring because phosphate is one of the nutrients that result in algal blooms. As with other nutrients, seamless monitoring of phosphate is important for understanding and evaluating eutrophication. However, field-deployable phosphate sensors have not been well developed yet due to the chemical characteristics of phosphate. In this paper, we report on a luminescent coordination polymer particle (CPP) that can respond selectively and sensitively to a phosphate ion against other ions in an aquatic ecosystem. The CPPs with an average size of $88.1 \pm 12.2 \mathrm{~nm}$ are embedded into membranes for reusable purpose. Due to the specific binding of phosphates to europium ions, the luminescence quenching behavior of CPPs embedded into membranes shows a linear relationship with phosphate concentrations $(3-500 \mu \mathrm{M})$ and detection limit of $1.52 \mu \mathrm{M}$. Consistent luminescence signals were also observed during repeated measurements in the $\mathrm{pH}$ range of $3-10$. Moreover, the practical application was confirmed by sensing phosphate in actual environmental samples such as tap water and lake water.
\end{abstract}

Keywords: coordination polymer particle; luminescence; quenching; phosphate sensor; environmental analysis

\section{Introduction}

Algal blooms are known to occur in lakes, rivers, and oceans, with complex behavior due to various prevailing factors such as nutrients $\left(\mathrm{NO}_{3}{ }^{-}, \mathrm{NH}_{4}{ }^{+}\right.$, and $\left.\mathrm{PO}_{4}{ }^{3-}\right)$ along with water temperature, flow rate, rainfall, and dissolved oxygen [1]. In particular, harmful algal blooms (HABs) of Microcystis, Anabaena, Oscillatoria, and Aphanizomenon produce toxins and have a malignant effect on aquatic life and humans [2,3]. Extensive research has been conducted on the conservation of aquatic environments through seamless data monitoring of HABs, prediction of their occurrence, and intervention of appropriate algaeremoval treatments [4]. To this end, water environments have been monitored using various sensors such as $\mathrm{pH}$, chlorophyll a, phycocyanin, total nitrogen, $\mathrm{NH}_{4}-\mathrm{N}, \mathrm{NO}_{3}-\mathrm{N}$, total phosphorous (TP), and $\mathrm{PO}_{4}-\mathrm{P}$ sensors. Most sensors can be deployed in the field and applied seamlessly to gather signals using an Internet-of-Things platform. However, in the case of TP and $\mathrm{PO}_{4}-\mathrm{P}$, there is a general method for quantifying the reaction with a coloring agent after reacting with an oxidizing agent under the conditions of high temperature and high pressure. This complicated analysis process limits the field deployment applications of phosphate sensors without the aid of chemical reagents and makes phosphate sensors only applicable in the laboratory. Phosphate sensors have been proposed and devised for working with various mechanisms such as solid electrochemical redox types [5], liquid membrane-ion selective electrodes (ISE) [6], solid-state ISE [7,8], spectrophotometric, and 
photoluminescence types [9-11]. However, there are several difficulties in developing phosphate sensors. Firstly, phosphate ions prefer different forms according to the acidity of the dissolved aqueous solution. This makes it difficult to realize phosphate sensors that can work in a wide $\mathrm{pH}$ range. Secondly, the widely adapted size-exclusion principle for sensor selectivity is difficult to apply in phosphate sensors owing to its relatively large ion size. Furthermore, it is hard to implement a phosphate sensor using non-covalent interactions such as hydrogen bonding as it has a large solvation energy according to the Hofmeister series [12]. Although ISE sensors have been studied for more than 45 years and show high performance for many cations and anions $\left(\mathrm{NH}_{4}^{+}, \mathrm{K}^{+}, \mathrm{Na}^{+}, \mathrm{Mg}^{2+}, \mathrm{Ca}^{2+}, \mathrm{Cl}^{-}, \mathrm{NO}_{3}{ }^{-}, \mathrm{CN}^{-}\right.$, and $\mathrm{F}^{-}$) [13], no ISE-type phosphate sensor with sufficient reliability in field applications has been developed. Note that it is necessary to detect the concentration of $\mathrm{PO}_{4}-\mathrm{P}$ at the level of $0.1 \mathrm{mg} / \mathrm{L}(3.2 \mu \mathrm{M})$, being an indicative level for probable problematic algal growth [14]. This detection level of phosphate is accomplished using spectrophotometric or photoluminescent analysis in an experimental setup consisting of complex fluid control devices and chemical storage/supplies [15].

Research areas on coordination polymer particles (CPPs) are expanding rapidly to various applications such as gas storage, catalysis, molecular sensing, bio-imaging, and drug delivery due to their significantly large surface area and tunability $[16,17]$. Our research group has made many efforts to develop diverse CPPs by using rationally designed functional building blocks $[18,19]$. Surface modification and control of size and shape over CPPs are considered critical for practical applications because a subtle change in the interface alters their physical and chemical properties [20]. Recently, several investigations have been also reported on the applications of phosphate sensors based on metal-organic frameworks $[9,21-24]$. In this study, to develop a phosphate sensor that can be easily deployed in the field and can be operated unattended, we present a photoluminescence phosphate sensor based on an CPP with high sensitivity, selectivity, and wide working range of $\mathrm{pH}$. The strong coordination interaction between europium and phosphate in the $\mathrm{CPP}$ is considered to provide exceptional selectivity and sensitivity as well as stability in various $\mathrm{pH}$ (Figure 1a). The CPP in this study is more durable in the aquatic environment than common luminescent metal-organic frameworks and can be synthesized in the form of nanoparticles that can be easily embedded into the porous membrane $[25,26]$. The resulting luminescent membrane containing the CPP is expected to be installed in a probe-type photoluminescent sensor adapted for the commercial dissolved-oxygen sensor as shown in Figure $1 b$ [27]. 
(a)
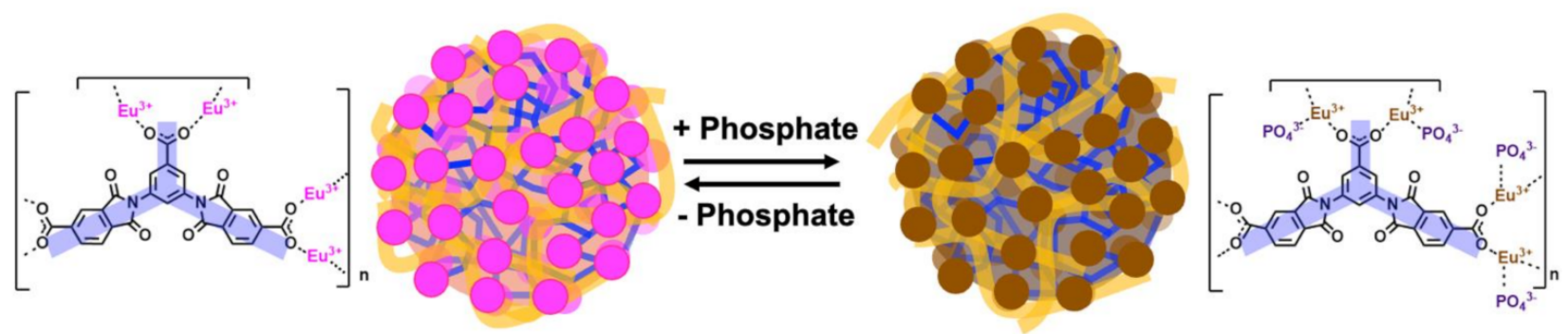

Luminescent Eu Quenched Eu

Multidentate ligand

Polymer

(b)

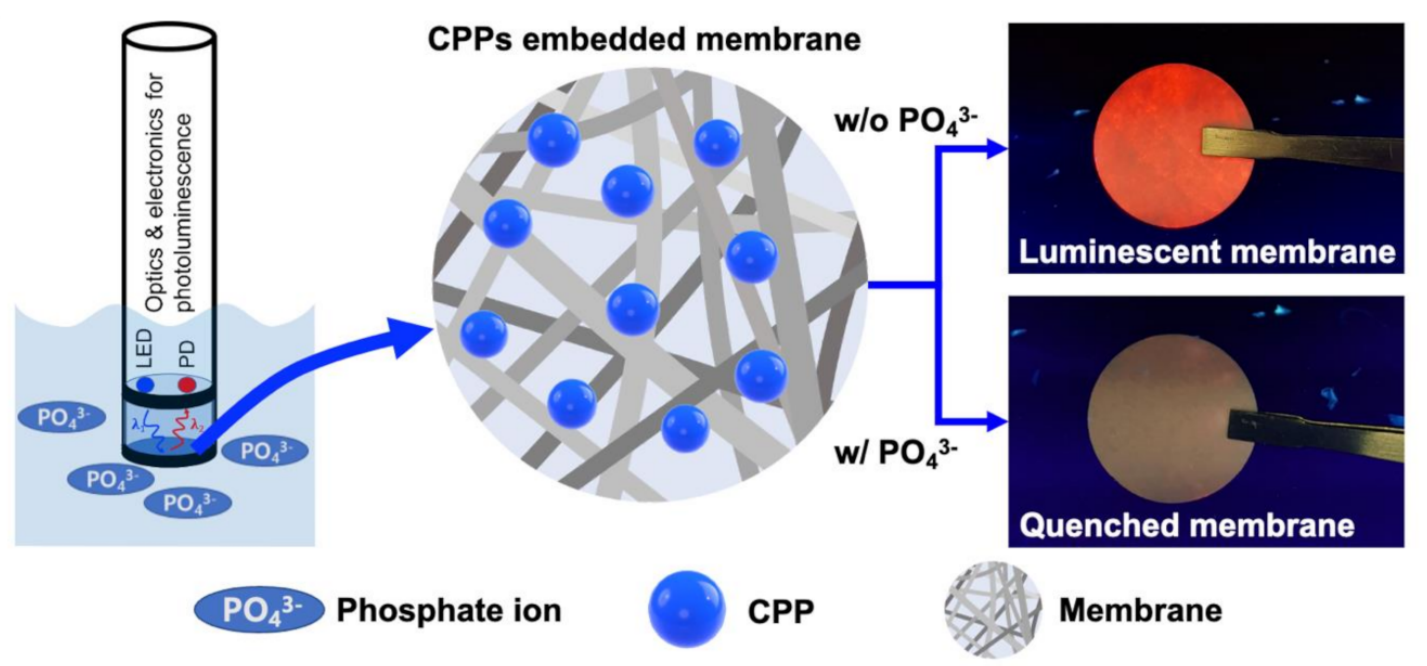

Figure 1. (a) Schematic illustration of the luminescent "turn-off" sensing based on CPP for detection of phosphate. (b) Conceptual configuration of phosphate sensor composed of light source, detector, and membrane containing CPPs as sensing materials.

\section{Materials and Methods}

\subsection{Preparation of Luminescent CPP}

$\mathrm{EuCl}_{3} \cdot 6 \mathrm{H}_{2} \mathrm{O}$ was purchased from TCI (Tokyo, Japan), and polyvinylpyrrolidone (PVP) was bought from Thermo Scientific ${ }^{\mathrm{TM}}$ (Massachusetts, USA). Other reagents such as dimethylformamide (DMF), ethanol, potassium phosphate and $\mathrm{H}_{2} \mathrm{SO}_{4}$ were purchased from Samchun Chemicals (Pyeongtaek, Korea). 2,2'-(5-Carboxy-1,3-phenylene)bis(1,3dioxoisoindoline-5-carboxylic acid) (TCA) was synthesized according to a previously reported method with modification [28]. Eu-TCAs composed of Eu ions and TCA as a multidentate ligand containing three carboxylic groups were synthesized in the form of $\mathrm{CPP}$ under solvothermal and high-pressure conditions. $\mathrm{EuCl}_{3} \cdot 6 \mathrm{H}_{2} \mathrm{O}(235 \mathrm{mg}, 0.64 \mathrm{mmol}$, 5.3 equation), TCA (60 mg, $0.12 \mathrm{mmol}, 1$ equation), and PVP (Avg. Mol. Wt. $=50,000$, $1600 \mathrm{mg}$ ) were mixed in DMF $(32 \mathrm{~mL})$ and ethanol $(19 \mathrm{~mL})$. The reaction mixture was acidified by adding $\mathrm{H}_{2} \mathrm{SO}_{4}(33.3 \mu \mathrm{L})$ and then sonicating for $30 \mathrm{~min}$ to obtain a clear solution. A hydrothermal reactor was charged with the reaction mixture, and a solvothermal reaction condition was applied at $150{ }^{\circ} \mathrm{C}$ for $12 \mathrm{~h}$. The reaction mixture was cooled over the next $6 \mathrm{~h}$, and colloidal particles were precipitated by centrifugation. Three cycles of wash with dry DMF and centrifugation were followed by a final wash with ethanol. After drying at $70^{\circ} \mathrm{C}$ for $3 \mathrm{~h}$, a pinkish-white solid was obtained. 


\subsection{Preparation of Eu-TCA Embedded in Glass Microfiber Filters (Eu-TCA/GMF)}

GMFs with a pore size of $1.2 \mu \mathrm{m}$ were used as a membrane. First, the GMF was placed at the bottom of each well of a 96-well microplate. An Eu-TCA dispersion with a concentration of $0.5 \mathrm{wt} . \%$ was prepared and sonicated for $10 \mathrm{~min}$. Next, each well was filled with $60 \mu \mathrm{L}$ of the Eu-TCA dispersion and dried overnight. The microplate containing the Eu-TCA/GMF was washed with deionized water several times to remove unadsorbed Eu-TCA particles to the GMF.

\subsection{Phosphate Detection of Eu-TCA Dispersion}

In the study of the quenching of Eu-TCA dispersion by phosphate, $10 \mathrm{mg}$ of Eu-TCA powder was dispersed in $25 \mathrm{~mL}$ of deionized water. After ultrasonication for $20 \mathrm{~min}$, $2.5 \mathrm{~mL}$ of Eu-TCA stock solution and the different concentrations of phosphate solution $(2.5 \mathrm{~mL})$ were added to a test tube. The solution was thoroughly mixed and then incubated for $10 \mathrm{~min}$ before photoluminescence measurements.

\subsection{Characterization}

The surface morphology and microstructure of the Eu-TCA were analyzed using field-emission scanning electron microscopy (SEM, S-4800, Hitachi, Tokyo, Japan) and transmission electron microscopy (TEM, Tecnai G2 F30, FEI, USA) with energy dispersive X-ray spectroscopy (EDX). Powder X-ray diffraction (PXRD) data were collected using a Rigaku diffractometer (MiniFlex II, Rigaku, Japan). The diffraction pattern was measured with $\mathrm{Cu}-\mathrm{K} \alpha$ radiation $(\lambda=1.5418 \AA)$ with voltage of $30 \mathrm{kV}$ and current of $15 \mathrm{~mA}$. The $2 \theta$ range was $5-50^{\circ}$. Fourier transform infrared (FTIR) spectra were obtained using a Nicolet iS10 FTIR spectrometer (Thermo Fisher Scientific, Waltham, MA, USA), and X-ray photoelectron spectroscopy (XPS) measurements were performed by using a K-Alpha ${ }^{+}$ spectrometer (Thermo Fisher Scientific, USA) equipped with a micro-focused monochromatic Al K $\alpha$ X-ray source. The photoluminescence of the Eu-TCA dispersion was studied using a spectrophotometer (FL 6500, PerkinElmer, Waltham, MA, USA). To investigate the phosphate sensing performance of the Eu-TCA/GMF, the luminescence intensity of the microplate containing the Eu-TCA/GMF was measured by using a microplate reader (Infinite M200, TECAN, Swiss, Basel, Switzerland). The excitation and emission wavelengths were selected as 260 and $615 \mathrm{~nm}$, respectively.

\section{Results and Discussion}

\subsection{Morphology and Structure Analysis}

The Eu-TCA was successfully obtained by controlling the synthetic parameters: the molar ratio between $\mathrm{Eu}^{3+}$ ions and ligands, concentration, reaction temperature, and $\mathrm{pH}$. PVP addition and $\mathrm{pH}$ control affected the shape and crystallinity of CPPs. PVP contains pyrrolidone groups along their backbone, and the carbonyl groups on pyrrolidones weakly bind to metal ions. These additional coordination bonds interrupt the bonds between $\mathrm{Eu}^{3+}$ ions and TCA ligands, leading to an irregular arrangement of components around the surface region. They also play an important role in controlling the size of particles. TCA ligand molecules and metal ions concentrate around the PVP molecule even in the early state and at lower $\mathrm{pH}$ conditions. For this reaction, an appropriate amount of PVP relative to the amounts of Eu ions and TCA ligands should be used to achieve size and shape control. When the amount of PVP was reduced to one-third of the synthetic condition as described in Materials and Methods, the Eu-TCA was obtained as anisotropic rice-shaped particles (Supplementary Figure S1).

It is important to prepare homogeneous CPP dispersion in that it ensures batch-tobatch reproducibility and thus provides reliable detection of phosphate. Size control was improved by adding sulfuric acid to the reaction mixture. This was used to delay the rate of $\mathrm{pH}$ elevation owing to decomposition of DMF into dimethyl amine. Kinetic control over $\mathrm{CPP}$ formation requires such $\mathrm{pH}$ adjustment at the early stage of the reaction or temperature control over the reaction time [29]. 
The morphology of the Eu-TCA was studied by SEM and TEM analysis. As shown in Figure 2a, the obtained Eu-TCA particles are spherical. The size distribution of the Eu-TCA was determined based on the SEM image by measuring the size of 334 particles. It was confirmed that the Eu-TCA exhibits normal distribution with an average size of $88.1 \pm 12.2 \mathrm{~nm}$ (Figure 2b). In addition, as shown in Figure 2c, the cumulative frequency distribution indicates that most of the Eu-TCAs are less than $101.7 \mathrm{~nm}\left(d_{90}\right)$. The morphology and size of the Eu-TCA from TEM observations were in good agreement with those in the SEM analysis and dynamic light scattering measurement (Figure $2 \mathrm{~d}$ and Supplementary Figure S2). The elemental composition of the CPPs was estimated by using EDX. As shown in Figure 2e, Eu, C, and $\mathrm{O}$ peaks appear as the main strong peaks, whereas $\mathrm{N}$ is a minor peak. This result indicated that a large portion of the CPP is a contribution of $\mathrm{Eu}^{3+}$ ions, and the remaining is shared by TCA ligands and PVP polymers. It is clear from the elemental mapping of the single Eu-TCA that $\mathrm{C}, \mathrm{O}, \mathrm{Eu}$, and $\mathrm{N}$ are uniformly distributed throughout the spherical particle (Figure $2 \mathrm{f}-\mathrm{i}$ ). PXRD measurements of the particles exhibited no strong diffraction peaks (Figure $2 \mathrm{j}$ ). These results indicated the formation of amorphous coordination polymers due to the premature short-range regularity.
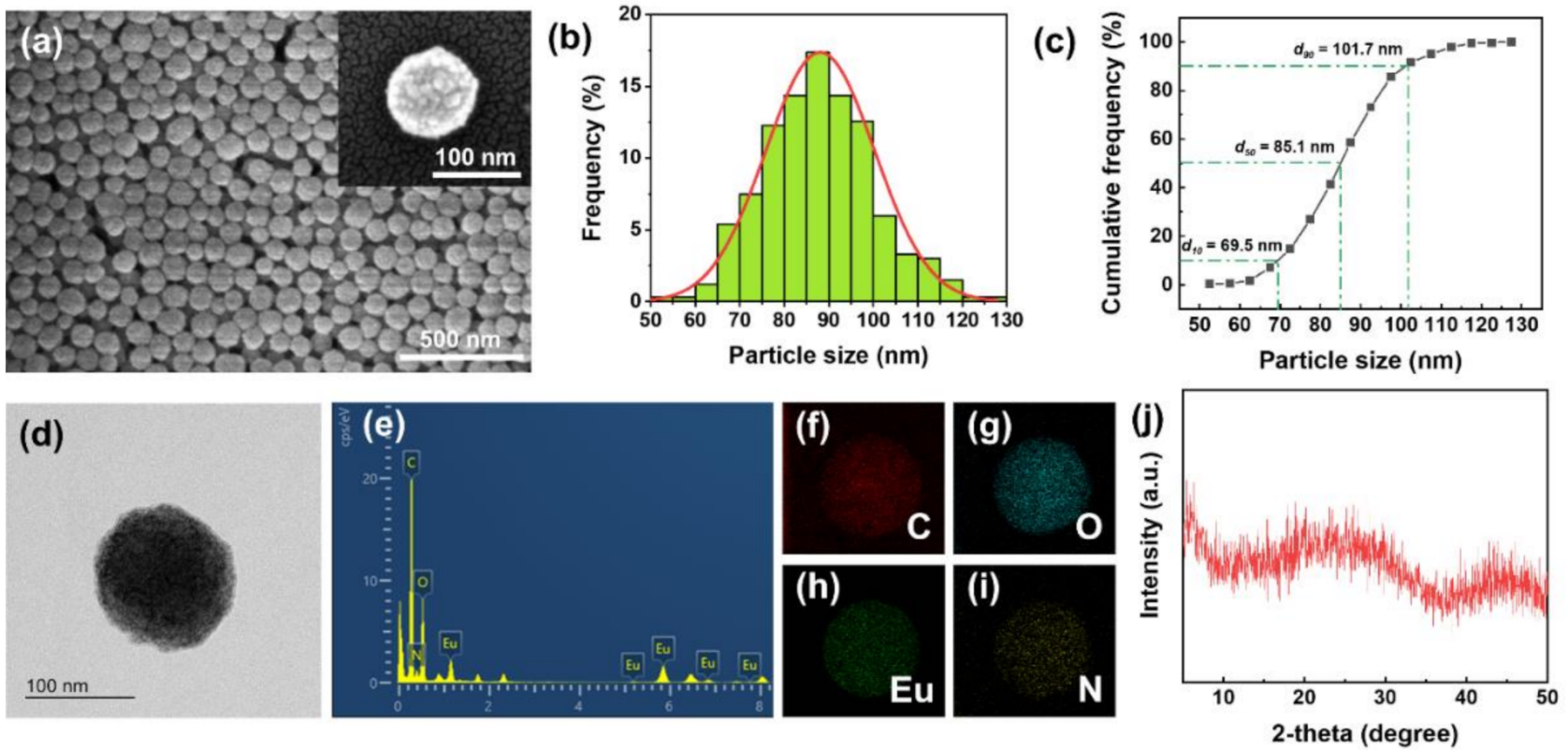

Figure 2. (a) SEM images, (b) size histogram, (c) cumulative frequency distribution, (d) TEM image, (e) EDX spectrum, (f-i) EDX elemental mapping, and (j) XRD spectrum of Eu-TCA.

\subsection{Evaluation of Phosphate Sensing Materials Based on Luminescence Quenching}

Figure 3 a shows the excitation and emission spectra of the Eu-TCA dispersion in deionized water $(200 \mathrm{mg} / \mathrm{L})$. The excitation spectrum was measured at an emission wavelength of $615 \mathrm{~nm}$, and the emission spectrum was determined under excitation at $260 \mathrm{~nm}$. The broad band in the range of $230-300 \mathrm{~nm}$ in the excitation spectrum results from the $\mathrm{O}^{2-} \rightarrow$ $\mathrm{Eu}^{3+}$ charge transfer and $\pi-\pi^{*}$ electronic transition in coordinated organic ligands [30-32]. As shown in Figure $3 a$ and Supplementary Figure S3, the emission spectra exhibit five characteristic transitions of $\mathrm{Eu}^{3+}$ centered at 579,591, 615, 651, and $699 \mathrm{~nm}$, corresponding to the ${ }^{5} \mathrm{D}_{0} \rightarrow{ }^{7} \mathrm{~F}_{J}$ transitions $(J=0,1,2,3$, and 4$)$, respectively [33]. 

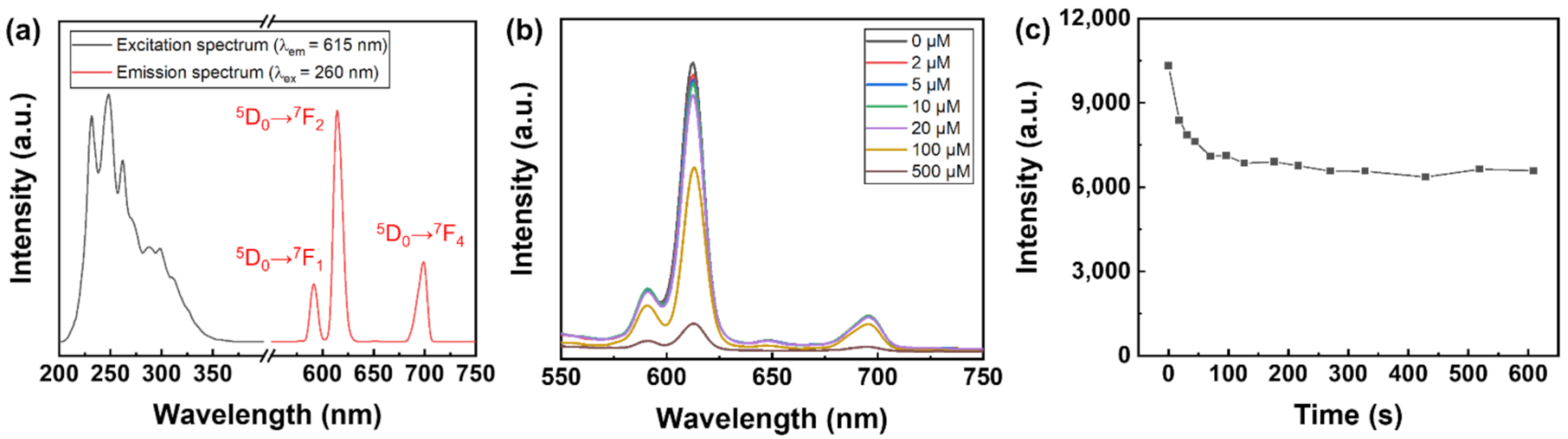

Figure 3. (a) Photoluminescence spectra of Eu-TCA dispersion. (b) Luminescence spectra of EuTCA dispersion in the presence of different amount of phosphate under the excitation at $260 \mathrm{~nm}$. (c) Time-dependent luminescence intensity of Eu-TCA dispersion upon the addition of $100 \mu \mathrm{M}$ of phosphate.

To investigate the detection performance of the Eu-TCA for phosphate, luminescence titration experiments were conducted at various concentrations of phosphate. Luminescence quenching occurred upon the addition of phosphate, and the luminescence intensity decreased with the increase in phosphate concentration (Figure 3b). The efficiency of the luminescence quenching process can be determined by the following Stern-Volmer equation:

$$
I_{0} / I=1+K_{S V} \times[Q],
$$

where $I$ and $I_{0}$ are the luminescence intensities with and without the quencher, respectively, $K_{\mathrm{SV}}$ is the quenching constant, and $[Q]$ is the molar concentration of the quencher. Supplementary Figure $S 4$ shows the Stern-Volmer plot for the ${ }^{5} \mathrm{D}_{0} \rightarrow{ }^{7} \mathrm{~F}_{2}$ transition at $615 \mathrm{~nm}$ for the Eu-TCA dispersion. A good linear Stern-Volmer relationship between the ratio of luminescence intensity $\left(I_{0} / I\right)$ and concentrations ranging from 2 to $100 \mu \mathrm{M}$ was observed with a $K_{\mathrm{SV}}$ of $5.78 \times 10^{3} \mathrm{M}^{-1}$. While $I_{0} / I$ increased as phosphate concentrations increased, the ratio of lifetime $\left(\tau_{0} / \tau\right)$ was almost unchanged in the $0-100 \mu \mathrm{M}$ phosphate concentrations in time-resolved photoluminescence experiments. On the contrary, both $I_{0} / I$ and $\tau_{0} / \tau$ increased as phosphate concentrations increased in the $100-500 \mu \mathrm{M}$ phosphate concentrations (Supplementary Figure S4). This indicates the simultaneous existence of static and dynamic quenching phenomena [34]. The response time of the Eu-TCA dispersion was found to be $123 \mathrm{~s}$ from the time-dependent studies (Figure 3c).

As shown in Figure 4, the formation of the Eu-TCA and its phosphate-binding event was monitored by using FTIR. The TCA ligand exhibited a strong $\mathrm{C}=\mathrm{O}$ stretching peak at $1730 \mathrm{~cm}^{-1}$, which was red-shifted to $1720 \mathrm{~cm}^{-1}$ after the formation of the Eu-TCA. The asymmetric $1599 \mathrm{~cm}^{-1}$ peak from the carboxylic groups grew bigger and red-shifted to $1557 \mathrm{~cm}^{-1}$. The $\mathrm{CH}_{2}$ stretching vibration at $1386 \mathrm{~cm}^{-1}$ and the imide ring deformation peak at $1098 \mathrm{~cm}^{-1}$ increased due to the formation of coordination polymers with PVP. The carbonyl peak at $1657 \mathrm{~cm}^{-1}$ overlapped with the imide carbonyl peak, which originated from PVP. After incubation with phosphate, the intensities of the peaks at 1386 and $1098 \mathrm{~cm}^{-1}$ significantly decreased, indicating that the features of PVP considerably diminished. Two new peaks appeared at 1062 and $1014 \mathrm{~cm}^{-1}$. These corresponded to coordinated $\mathrm{P}-\mathrm{O}$ bonds in two different binding modes [35]. Overall changes in the FTIR data strongly supported the binding of phosphate and rearrangement of coordination bonds around $\mathrm{Eu}^{3+}$ metal centers included in Eu-TCAs. 


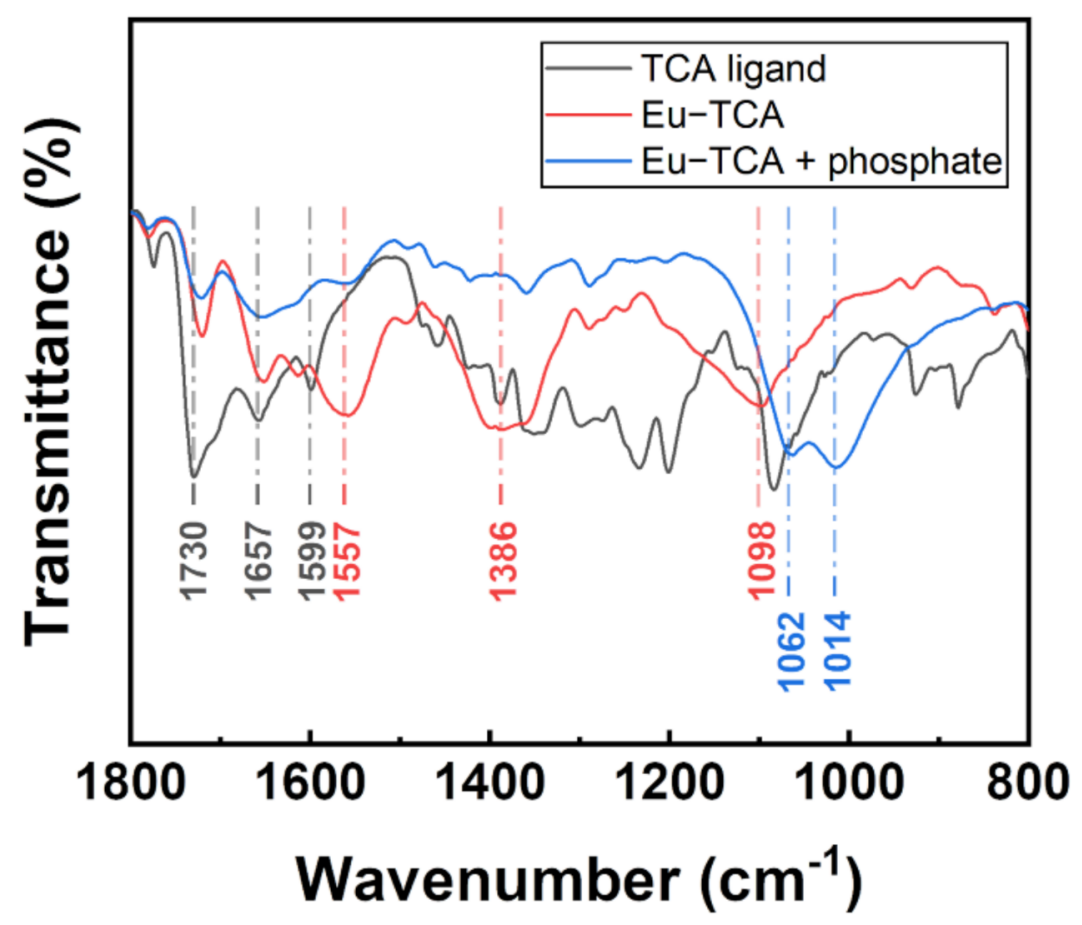

Figure 4. FTIR spectra of the TCA ligand and Eu-TCA before and after incubation with phosphate.

XPS analysis was conducted to further investigate the coordination interactions between the Eu-TCA and phosphate. As shown in Figure 5a, the characteristic peaks corresponding to $\mathrm{Eu}, \mathrm{O}$, and $\mathrm{C}$ are observed from the Eu-TCA before and after incubation with phosphate. The peak at $190.1 \mathrm{eV}$, assigned to P 2s, indicates that phosphate ions are successfully introduced into the Eu-TCA as shown in Figure 5a (curve II). Figure 5b shows the Eu 3d spectra of the Eu-TCA before and after incubation with phosphate. The binding energies at 1162.9 and $1133.3 \mathrm{eV}$ in Figure $5 \mathrm{~b}$ (curve I) are assigned to $\mathrm{Eu} 3 \mathrm{~d}_{3 / 2}$ and Eu $3 \mathrm{~d}_{5 / 2}$, respectively, originating from the Eu-O clusters coordinated with carboxylic groups in the TCA ligands of the Eu-TCA. Similar to previous reports, the peaks of Eu $3 d_{3 / 2}$ and $\mathrm{Eu} 3 \mathrm{~d}_{5 / 2}$ were shifted toward higher binding energies after the inclusion of phosphate into the Eu-TCA framework as shown in Figure 5b (curve II) [24,36]. The shifts in the binding energy were caused by replacing carboxylic groups coordinated with Eu-O clusters with phosphoric groups. The interaction between $\mathrm{Eu}^{3+}$ ions and more electronegative $\mathrm{P}-\mathrm{O}$ bonds resulted in the loss of electron density in Eu. Consequently, the binding energies of electrons in $\mathrm{Eu} 3 \mathrm{~d}$ increased. The $\mathrm{O} 1 \mathrm{~s}$ spectrum of the Eu-TCA was deconvoluted into four components positioned at 532.6, 531.8, 531.1, and 529.6 eV (Figure S5a). The peak at $529.6 \mathrm{eV}$ represented lattice $\mathrm{O}$ bound to $\mathrm{Eu}(\mathrm{Eu}-\mathrm{O}-\mathrm{Eu})$. The other three peaks were related to water molecules or hydroxyl functional groups on the sample: the $\mathrm{OH}$ group with oxygen at the bridging oxygen site (bridging $\mathrm{OH}, 531.1 \mathrm{eV}$ ), the $\mathrm{OH}$ group with oxygen attached to Eu forming Eu-OH groups (terminal $\mathrm{OH}, 531.8 \mathrm{eV}$ ), and adsorbed water molecules on the sample surface $(532.6 \mathrm{eV})$. After interacting with phosphate, the ratio of the components positioned at 532.6 and $531.8 \mathrm{eV}$ to $\mathrm{O} 1 \mathrm{~s}$ spectrum increased from $15.4 \%$ and $30.3 \%$ to $18.7 \%$ and $40.7 \%$, respectively, whereas the ratio of the components peaked at 531.1 and $529.6 \mathrm{eV}$ to $\mathrm{O} 1 \mathrm{~s}$ spectrum decreased from $47.1 \%$ and $7.2 \%$ to $35.9 \%$ and $4.7 \%$, respectively (Figure S5a,b). This phenomenon can be attributed to the formation of Eu-O-P bonds and the replacement of $\mathrm{OH}$ groups by phosphate [36]; this is consistent with the results of the FTIR analysis presented in Figure 4. 

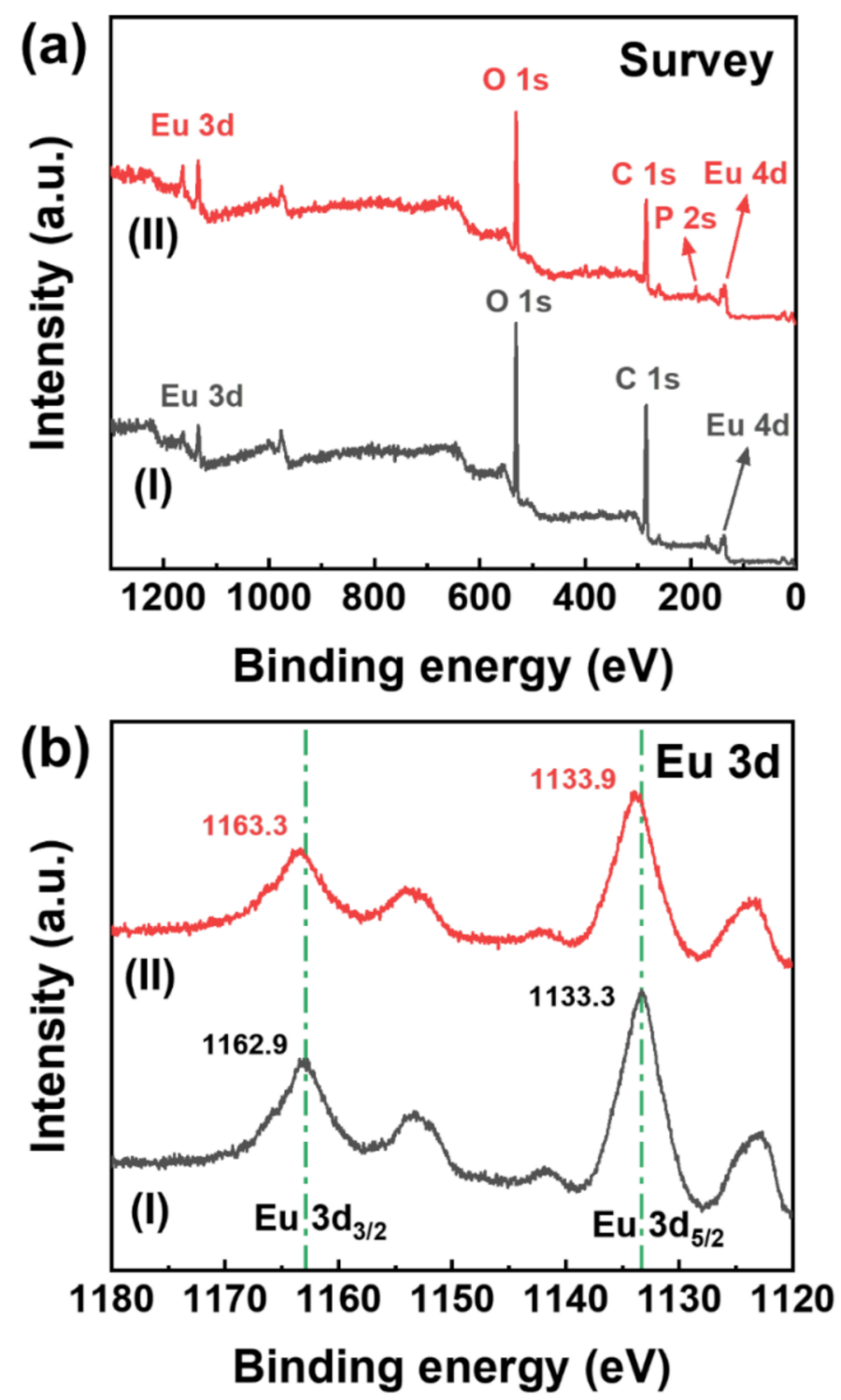

Figure 5. XPS spectra of Eu-TCA: (a) survey spectra of Eu-TCA before (I) and after (II) incubation with phosphate. (b) Eu 3d XPS spectra before (I) and after (II) incubation with phosphate.

\subsection{Phosphate Sensing Properties of Eu-TCA/GMF}

For practical use, the sensing materials are recommended to be embedded in solid substrates because the recycling process of suspension in which the sensing material is homogeneously dispersed is complex and time-consuming. In this study, we propose the Eu-TCA/GMF as a reusable phosphate sensor. As shown in Figure 6a, the Eu-TCA nanoparticles are densely embedded on the surface of the microfiber over the entire filter, despite the washing process described in Materials and Methods. In order to evaluate the batch-to-batch reproducibility of the Eu-TCA/GMF, the luminescence intensities of the independently fabricated Eu-TCA/GMFs were investigated as shown in Figure 6b. The emission at $615 \mathrm{~nm}$ was monitored at an excitation wavelength of $260 \mathrm{~nm}$, similar to the experiments in the Eu-TCA dispersion. The relative standard deviation of the luminescence intensity was $2.5 \%$, which indicates that the batch-to-batch fabrication of the Eu-TCA/GMF is reproducible. 

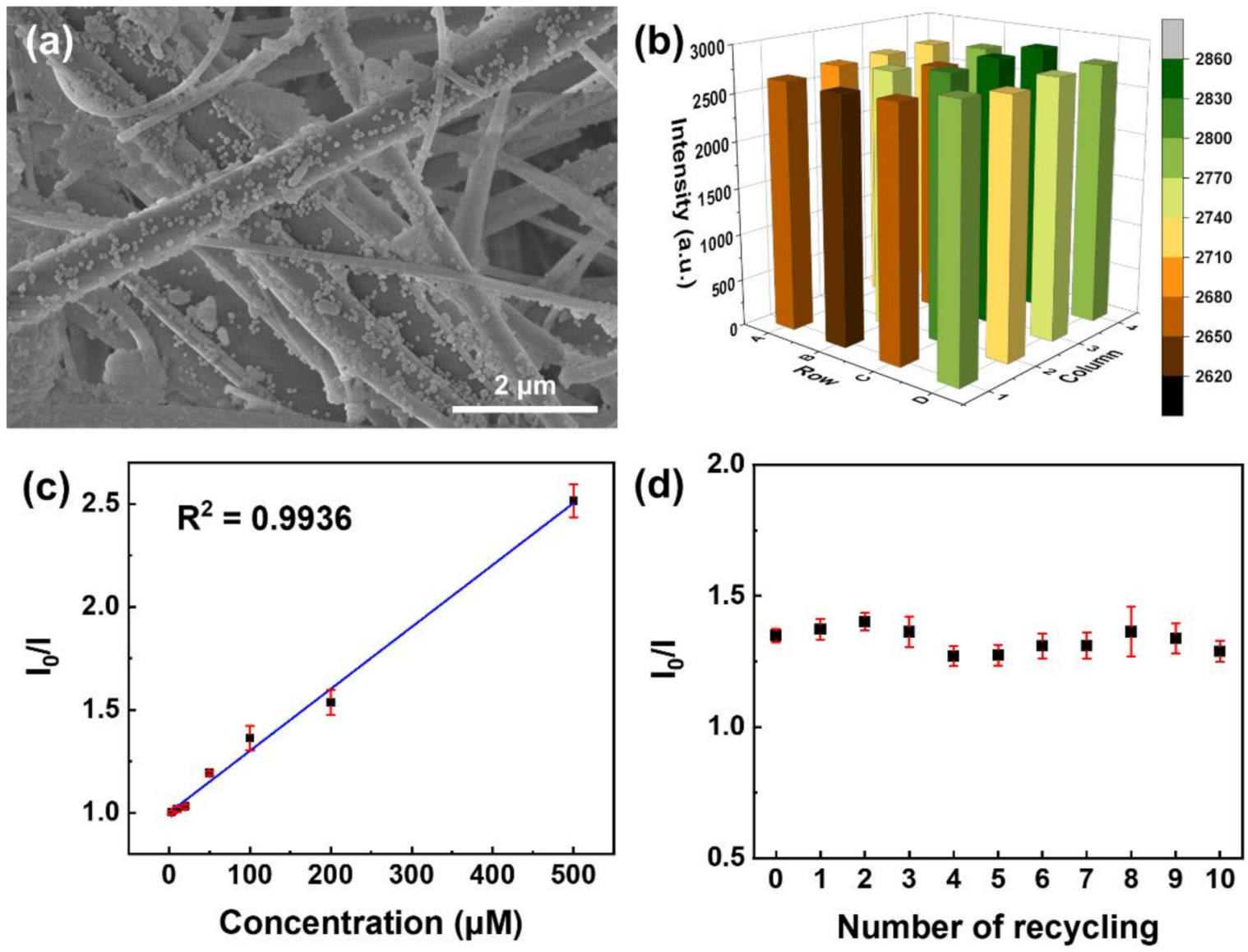

Figure 6. (a) SEM image of Eu-TCA/GMF. (b) Luminescence intensity of $4 \times 4$ wells of microplate containing the independently fabricated Eu-TCA/GMFs. (c) Stern-Volmer plot of the luminescence quenching of Eu-TCA/GMF $(n=7)$ as a function of phosphate concentration. (d) The luminescence intensity of Eu-TCA/GMF $(n=7)$ after ten runs of recycling. The concentration of phosphate is $100 \mu \mathrm{M}$.

To measure the luminescence intensity of the Eu-TCA/GMF under exposure to phosphate anions, the wells of the microplate containing the Eu-TCA/GMF were filled with $200 \mu \mathrm{L}$ of phosphate solutions at concentrations ranging from 0 to $500 \mu \mathrm{M}$. Figure $6 \mathrm{c}$ shows $I_{0} / I$ as a function of phosphate concentration, where $I$ and $I_{0}$ denote the luminescence intensities of the Eu-TCA/GMF in deionized water $(200 \mu \mathrm{L})$ with and without phosphate, respectively. Upon the addition of phosphate to the wells of the microplate, the luminescence intensity of the Eu-TCA/GMF decreased. In addition, the phosphate detection of the Eu-TCA/GMF showed a linear response from 3 to $500 \mu \mathrm{M}\left(\mathrm{R}^{2}=0.9936\right)$ with a $K_{\mathrm{SV}}$ of $3.01 \times 10^{3} \mathrm{M}^{-1}$. A short response time (123 s) was observed from the Eu-TCA dispersion, whereas the response times of the Eu-TCA/GMF were $982 \mathrm{~s}$ at $100 \mu \mathrm{M}$ (Figure 3c and Supplementary Figure S6). The slow response of the Eu-TCA/GMF can be attributed to the limited effective surface and low reaction probability of Eu-TCA for the interaction with phosphate in the membrane. The limit of detection (LOD) was derived from the $3 \sigma$ IUPAC criteria using the following equation:

$$
\mathrm{LOD}=3 \sigma / k,
$$

where $\sigma$ is the standard deviation and $k$ is the slope $[37,38]$. The standard deviation was calculated by using multiple luminescence intensities of the Eu-TCA/GMF in the absence of phosphate (blank). The slope was taken from the linear fit curve for luminescence intensity against phosphate concentration as shown in Figure S7. The LOD of the Eu- 
TCA/GMF was determined to be $1.52 \mu \mathrm{M}$, which is below the indicative level $(3.23 \mu \mathrm{M})$ for probable problematic algal growth [14]. As shown in Supplementary Table S1, the sensing performance of the Eu-TCA/GMF was comparable or better than those of the other methods for phosphate detection in terms of LOD.

To study the performance of reused Eu-TCA/GMF, the wells of a microplate containing the Eu-TCA/GMF were incubated with an aqueous phosphate solution of $100 \mu \mathrm{M}$ for complete interaction of phosphate with the Eu-TCA. Then, the microplate was washed several times with deionized water. After ten runs of the recycling process, $I_{0} / I$ barely changed, indicating that the Eu-TCA/GMF can act as a reusable sensing material with high repeatability for detecting phosphate (Figure $6 \mathrm{~d}$ ).

The effects of $\mathrm{pH}$ on the luminescence intensity of the Eu-TCA/GMF were studied. The wells of the microplate containing the Eu-TCA/GMF were filled with $200 \mu \mathrm{L}$ of solutions in the $\mathrm{pH}$ range of $2-10$. Figure $7 \mathrm{a}$ shows the luminescence intensity of the Eu-TCA/GMF under various $\mathrm{pH}$ conditions as a function of time. The luminescence intensity of the Eu-TCA/GMF at pH 2 showed a time-dependent decrease, whereas the luminescence intensity in the $\mathrm{pH}$ range of 3-10 was stable over time (40 $\mathrm{min})$. To explore the recovery of the luminescence intensity, the Eu-TCA/GMF was washed with deionized water, and the luminescence intensity was measured with $200 \mu \mathrm{L}$ of a $\mathrm{pH} 7$ solution. Figure $7 \mathrm{~b}$ shows $I_{\text {after }} / I_{\text {before }}$ of the Eu-TCA/GMF, where $I_{\text {before }}$ is the luminescence intensity at pH 7 before exposure to the $\mathrm{pH}$ solution and $I_{\text {after }}$ is the luminescence intensity at $\mathrm{pH} 7$ after exposure to the $\mathrm{pH}$ solution. Except for $I_{\text {after }} / I_{\text {before }}$ of the Eu-TCA/GMF exposed to a $\mathrm{pH} 2$ solution, the luminescent intensity was almost unchanged in the $\mathrm{pH}$ range of 3-10, suggesting that the Eu-TCA/GMF can be used in a wide range of $\mathrm{pH}$ conditions for detecting phosphate. In the case of other fluorescence-based phosphate sensing materials, a stable signal in such a wide $\mathrm{pH}$ range is not achieved (Supplementary Table S1). In this study, it is judged that $\mathrm{pH}$ stability was secured by forming a more stable CPP with the introduction of multidentate ligand containing three carboxylate functional groups.

(a)

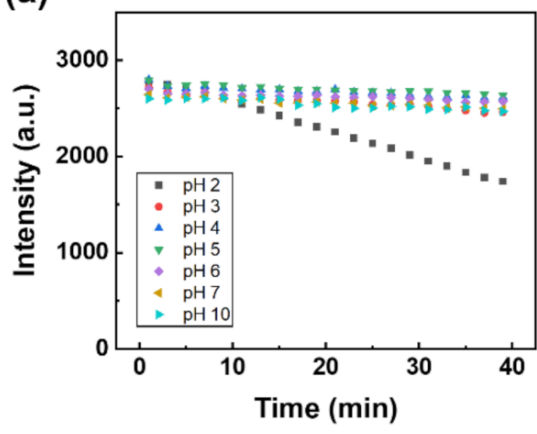

(b)

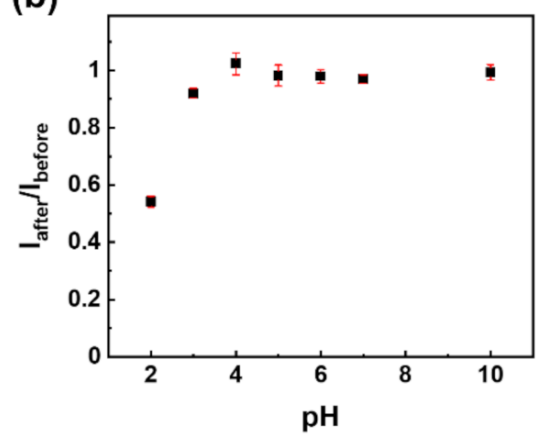

(c)

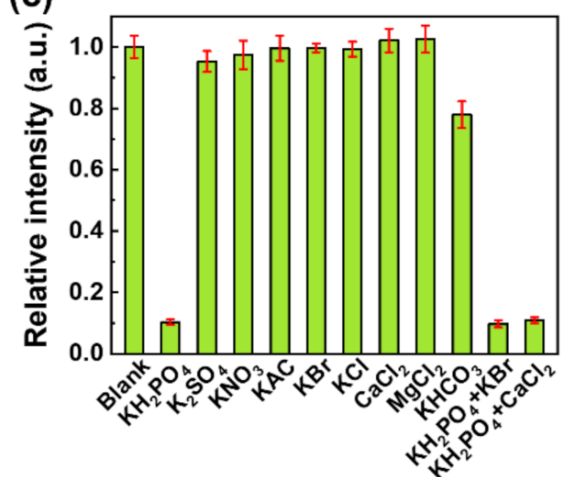

Figure 7. (a) The luminescence intensity of Eu-TCA/GMF as a function of time under various $\mathrm{pH}$ conditions. (b) $I_{\text {after }} / I_{\text {before }}$ of Eu-TCA/GMF $(n=6)$ as a function of $\mathrm{pH}$, where $I_{\text {before }}$ is the luminescence intensity before exposure to the $\mathrm{pH}$ solution and $I_{\text {after }}$ is the luminescence intensity after exposure to the $\mathrm{pH}$ solution. (c) The luminescence intensity of Eu-TCA/GMF $(n=6)$ immersed in phosphate and other various ionic solutions $(1 \mathrm{mM})$.

Selective detection is crucial for applying phosphate monitoring in aquatic ecosystems, where various types of ions exist. To study the selectivity of the Eu-TCA toward phosphate, the luminescence intensity of the $\mathrm{Eu}-\mathrm{TCA} / \mathrm{GMF}$ was compared with various ions, including $\mathrm{SO}_{4}{ }^{2-}, \mathrm{NO}_{3}{ }^{-}, \mathrm{CH}_{3} \mathrm{COO}^{-}, \mathrm{Br}^{-}, \mathrm{Cl}^{-}, \mathrm{HCO}_{3}{ }^{-}, \mathrm{K}^{+}, \mathrm{Mg}^{2+}$, and $\mathrm{Ca}^{2+}$, which usually exist in aquatic environments. Figure $7 \mathrm{c}$ shows the luminescence intensity upon exposure to various anion solutions $(1 \mathrm{mM})$. The addition of phosphate resulted in a drastic luminescence quenching effect. Except that the relative intensity in the presence of $\mathrm{HCO}_{3}{ }^{-}$ion was 0.8 , no obvious change in luminescence intensity was observed for the other ions. The 
effects of coexisting ions on the luminescence intensity were also investigated. The molar concentrations of phosphate and interfering analytes $\left(\mathrm{KBr}\right.$ or $\left.\mathrm{CaCl}_{2}\right)$ were $1 \mathrm{mM}$ and $3 \mathrm{mM}$, respectively. Although the concentration of interfering analyte is three times higher, the detection of phosphate was not affected by other ions, indicating an exceptional recognition capability of the Eu-TCA for phosphate in an aqueous solution.

To investigate the reliability of the Eu-TCA/GMF in practical applications, the phosphate detection was tested in tap water and lake water from Daecheong Lake as representative real environmental samples. The water samples were spiked with known concentrations of the phosphate solution. As summarized in Table 1, the recoveries and the corresponding relative standard deviation values of the spiked samples were found in the range of $95-104 \%$ and $2-4 \%$, respectively, suggesting that the proposed sensing platform is feasible for the phosphate detection in real environmental samples.

Table 1. Comparison of phosphate detection in real water samples using the proposed Eu-TCA/GMF.

\begin{tabular}{cccc}
\hline Samples & Spiked $(\boldsymbol{\mu M})$ & $\begin{array}{c}\text { Measured }(\boldsymbol{\mu M}) \\
\text { (Mean } \pm \text { Standard } \\
\text { Deviation, } \boldsymbol{n}=3 \text { ) }\end{array}$ & $\begin{array}{c}\text { Recovery (\%) } \\
\text { (Mean } \pm \text { Standard } \\
\text { Deviation, } \boldsymbol{n}=\mathbf{3} \text { ) }\end{array}$ \\
\hline Tap water & 0 & not detected $^{1}$ & \\
& 20 & $20.82 \pm 0.58$ & $104.10 \pm 2.90$ \\
Lake water & 50 & $50.30 \pm 1.22$ & $100.60 \pm 2.44$ \\
(Daecheong Lake) & 0 & not detected ${ }^{1}$ & \\
& 20 & $19.77 \pm 0.46$ & $98.85 \pm 2.30$ \\
& 50 & $47.45 \pm 2.06$ & $94.90 \pm 4.12$ \\
\hline
\end{tabular}

${ }^{1}$ The determination of phosphate in unspiked samples was also confirmed by phosphomolybdenum blue spectrophotometric methods.

\section{Conclusions}

This study demonstrates that luminescent nanoparticles (Eu-TCAs) composed of europium and multidentate ligands can be synthesized as CPPs that respond to phosphate through specific coordination interactions between lanthanides and phosphates, as confirmed by IR spectroscopy and XPS analysis. We considered that selective interactions between europium and phosphates provide exceptional selectivity and sensitivity in the application of phosphate sensors, and CPP instead of crystalline metal-organic frameworks promotes chemical stability under various $\mathrm{pH}$ conditions, from acidic to basic environments, with the aid of PVPs as a coat, capping, and matrix material. The nanoparticles were embedded into the glass microfiber filter, and exhibited a detection limit of $1.52 \mu \mathrm{M}$. Furthermore, the Eu-TCA/GMF showed durability from $\mathrm{pH} 3$ to $\mathrm{pH} 10$ and selectivity to phosphate ion against potential interfering ions in aqueous environment. Based on this study, a membrane containing luminescent nanoparticles is expected to be installed in a probe-type photoluminescent sensor and operated without any supply of redox chemicals or any activation chemical process.

Supplementary Materials: The following are available online at https://www.mdpi.com/article/10 $.3390 /$ polym14010190/s1, Supplementary Figure S1: SEM image of Eu-TCA microcrystals, Supplementary Figure S2: Dynamic light scattering analysis of Eu-TCA, Supplementary Figure S3: Emission spectrum of Eu-TCA dispersion $\left(\lambda_{\mathrm{ex}}=260 \mathrm{~nm}\right)$. The inset shows enlarged emission spectrum, Supplementary Figure S4: Stern-Volmer plot for the luminescence quenching of Eu-TCA dispersion $(n=5)$ upon addition of different concentrations of phosphate. Inset shows the SternVolmer plot in the concentration range of 2-500 $\mu \mathrm{M}$, Supplementary Figure S5: Experimental fit performed on O 1s XPS spectra before (a) and after (b) incubation with phosphate, Supplementary Figure S6: Time-dependent luminescence intensity of the Eu-TCA/GMF upon the addition of $100 \mu \mathrm{M}$ of phosphate, Figure S7: The luminescence intensity of Eu-TCA/GMF ( $n=7)$ under various phosphate concentrations and their linear fit curve for the estimation of LOD, Table S1: Comparison of various analytical methods for phosphate detection. 


\begin{abstract}
Author Contributions: Conceptualization, D.Y.K., J.H. and H.-K.L.; methodology, D.Y.K., B.J., J.H. and H.-K.L.; validation, D.Y.K., D.G.K., J.H. and H.-K.L.; formal analysis, D.Y.K., J.H. and H.-K.L.; investigation, D.Y.K., D.G.K., B.J., Y.I.K., J.H. and H.-K.L.; resources, J.H. and H.-K.L.; data curation, D.Y.K., D.G.K., Y.I.K., J.H. and H.-K.L.; writing-original draft preparation, D.Y.K., D.G.K., B.J., Y.I.K., J.H. and H.-K.L.; writing-review and editing, D.Y.K., J.H. and H.-K.L.; visualization, D.Y.K., D.G.K., J.H. and H.-K.L.; supervision, J.H. and H.-K.L.; project administration, J.H. and H.-K.L.; funding acquisition, J.H. and H.-K.L. All authors have read and agreed to the published version of the manuscript.
\end{abstract}

Funding: This work was supported by the ICT R\&D program of MSIT/IITP (2018-0-00219, Spacetime complex artificial intelligence blue-green algae prediction technology based on direct-readable water quality complex sensor and hyperspectral image).

Institutional Review Board Statement: Not applicable.

Informed Consent Statement: Not applicable.

Data Availability Statement: Not applicable.

Conflicts of Interest: The authors declare no conflict of interest.

\title{
References
}

1. Worsfold, P.; McKelvie, I.; Monbet, P. Determination of Phosphorus in Natural Waters: A Historical Review. Anal. Chim. Acta 2016, 918, 8-20. [CrossRef] [PubMed]

2. O'Neil, J.M.; Davis, T.W.; Burford, M.A.; Gobler, C.J. The Rise of Harmful Cyanobacteria Blooms: The Potential Roles of Eutrophication and Climate Change. Harmful Algae 2012, 14, 313-334. [CrossRef]

3. Paerl, H.W.; Paul, V.J. Climate Change: Links to Global Expansion of Harmful Cyanobacteria. Water Res. 2012, 46, 1349-1363. [CrossRef]

4. Bertone, E.; Burford, M.A.; Hamilton, D.P. Fluorescence Probes for Real-Time Remote Cyanobacteria Monitoring: A Review of Challenges and Opportunities. Water Res. 2018, 141, 152-162. [CrossRef]

5. Patel, V.; Selvaganapathy, P.R. Enhancing the Sensitivity of Cobalt Based Solid-State Phosphate Sensor Using Electrical Pretreatment. Sens. Actuators B Chem. 2021, 349, 130789. [CrossRef]

6. Matveichuk, Y.; Rakhman'ko, E.; Akayeu, Y.; Stanishevskii, D. Ion-Selective Electrodes Based on Long-Chain Quaternary Ammonium Salts with Enhanced Steric Accessibility, and Their Application for Determination of Hydrophilic Double-Charged Inorganic Anion. Chem. Pap. 2018, 72, 731-739. [CrossRef]

7. Xu, K.; Kitazumi, Y.; Kano, K.; Shirai, O. Phosphate Ion Sensor Using a Cobalt Phosphate Coated Cobalt Electrode. Electrochim. Acta 2018, 282, 242-246. [CrossRef]

8. Huang, Y.; Ye, Y.; Zhao, G.; Wu, X.; Kan, Y.; Mur, L.; Han, J.; Qin, H. An All-Solid-State Phosphate Electrode with H3PO4 Doped Polyaniline as the Sensitive Layer. Int. J. Electrochem. Sci. 2017, 12, 4677-4691. [CrossRef]

9. Chandra Rao, P.; Mandal, S. Europium-Based Metal-Organic Framework as a Dual Luminescence Sensor for the Selective Detection of the Phosphate Anion and $\mathrm{Fe}^{3+}$ Ion in Aqueous Media. Inorg. Chem. 2018, 57, 11855-11858. [CrossRef]

10. Gao, M.; Tang, B.Z. Fluorescent Sensors Based on Aggregation-Induced Emission: Recent Advances and Perspectives. ACS Sens. 2017, 2, 1382-1399. [CrossRef]

11. Wu, H.; Tong, C. A Specific Turn-On Fluorescent Sensing for Ultrasensitive and Selective Detection of Phosphate in Environmental Samples Based on Antenna Effect-Improved FRET by Surfactant. ACS Sens. 2018, 3, 1539-1545. [CrossRef]

12. Tafesse, F. Detection Mechanisms of Phosphate Sensitive Electrodes. Synth. React. Inorg. Met.-Org. Nano-Met. Chem. 2015, 45, 1687-1692. [CrossRef]

13. Lrving, H.M.N.H.; Zettler, H.; Baudin, G.; Freiser, H.; Guilbault, G.G.; Menis, O.; Rice, N.M.; Robertson, A.J.B.; Docherty, A.C.; Fischer, W.; et al. Recommendations for Nomenclature of Ion-Selective Electrodes (Recommendations 1975). Pure Appl. Chem. 1976, 48, 127-132. [CrossRef]

14. Morais, I.P.A.; Tóth, I.V.; Rangel, A.O.S.S. An Overview on Flow Methods for the Chemiluminescence Determination of Phosphorus. Anal. Phosphorus Environ. Agric. Samples 2005, 66, 341-347. [CrossRef]

15. Clinton-Bailey, G.S.; Grand, M.M.; Beaton, A.D.; Nightingale, A.M.; Owsianka, D.R.; Slavik, G.J.; Connelly, D.P.; Cardwell, C.L.; Mowlem, M.C. A Lab-on-Chip Analyzer for in Situ Measurement of Soluble Reactive Phosphate: Improved Phosphate Blue Assay and Application to Fluvial Monitoring. Environ. Sci. Technol. 2017, 51, 9989-9995. [CrossRef]

16. Einkauf, J.D.; Clark, J.M.; Paulive, A.; Tanner, G.P.; de Lill, D.T. A General Model of Sensitized Luminescence in Lanthanide-Based Coordination Polymers and Metal-Organic Framework Materials. Inorg. Chem. 2017, 56, 5544-5552. [CrossRef]

17. Sakata, Y.; Furukawa, S.; Kondo, M.; Hirai, K.; Horike, N.; Takashima, Y.; Uehara, H.; Louvain, N.; Meilikhov, M.; Tsuruoka, T.; et al. Shape-Memory Nanopores Induced in Coordination Frameworks by Crystal Downsizing. Science 2013, 339, 193. [CrossRef] [PubMed] 
18. Jeon, Y.-M.; Heo, J.; Mirkin, C.A. Dynamic Interconversion of Amorphous Microparticles and Crystalline Rods in Salen-Based Homochiral Infinite Coordination Polymers. J. Am. Chem. Soc. 2007, 129, 7480-7481. [CrossRef] [PubMed]

19. Jeon, Y.-M.; Armatas, G.S.; Heo, J.; Kanatzidis, M.G.; Mirkin, C.A. Amorphous Infinite Coordination Polymer Microparticles: A New Class of Selective Hydrogen Storage Materials. Adv. Mater. 2008, 20, 2105-2110. [CrossRef]

20. Wang, S.; McGuirk, C.M.; d'Aquino, A.; Mason, J.A.; Mirkin, C.A. Metal-Organic Framework Nanoparticles. Adv. Mater. 2018, 30, 1800202. [CrossRef]

21. Das, A.; Das, S.; Trivedi, V.; Biswas, S. A Dual Functional MOF-Based Fluorescent Sensor for Intracellular Phosphate and Extracellular 4-Nitrobenzaldehyde. Dalton Trans. 2019, 48, 1332-1343. [CrossRef]

22. Asha, K.S.; Bhattacharjee, R.; Mandal, S. Complete Transmetalation in a Metal-Organic Framework by Metal Ion Metathesis in a Single Crystal for Selective Sensing of Phosphate Ions in Aqueous Media. Angew. Chem. Int. Ed. 2016, 55, 11528-11532. [CrossRef]

23. Fan, C.; Lv, X.; Tian, M.; Yu, Q.; Mao, Y.; Qiu, W.; Wang, H.; Liu, G. A Terbium(III)-Functionalized Zinc(II)-Organic Framework for Fluorometric Determination of Phosphate. Microchim. Acta 2020, 187, 84. [CrossRef] [PubMed]

24. Song, X.; Ma, Y.; Ge, X.; Zhou, H.; Wang, G.; Zhang, H.; Tang, X.; Zhang, Y. Europium-Based Infinite Coordination Polymer Nanospheres as an Effective Fluorescence Probe for Phosphate Sensing. RSC Adv. 2017, 7, 8661-8669. [CrossRef]

25. Spokoyny, A.M.; Kim, D.; Sumrein, A.; Mirkin, C.A. Infinite Coordination Polymer Nano- and Microparticle Structures. Chem. Soc. Rev. 2009, 38, 1218-1227. [CrossRef]

26. Vegas, V.G.; Villar-Alonso, M.; Gómez-García, C.J.; Zamora, F.; Amo-Ochoa, P. Direct Formation of Sub-Micron and Nanoparticles of a Bioinspired Coordination Polymer Based on Copper with Adenine. Polymers 2017, 9, 565. [CrossRef]

27. Wei, Y.; Jiao, Y.; An, D.; Li, D.; Li, W.; Wei, Q. Review of Dissolved Oxygen Detection Technology: From Laboratory Analysis to Online Intelligent Detection. Sensors 2019, 19, 3995. [CrossRef] [PubMed]

28. Fukubayashi, Y.; Nakai, M.; Yanaka. A Method for Producing Organic Compound. WO 2020/009016 A1, 9 January 2020.

29. Heravi, M.M.; Ghavidel, M.; Mohammadkhani, L. Beyond a Solvent: Triple Roles of Dimethylformamide in Organic Chemistry. RSC Adv. 2018, 8, 27832-27862. [CrossRef]

30. Gaspar, R.D.L.; Mazali, I.O.; Sigoli, F.A. Particle Size Tailoring and Luminescence of Europium(III)-Doped Gadolinium Oxide Obtained by the Modified Homogeneous Precipitation Method: Dielectric Constant and Counter Anion Effects. Colloids Surf. Physicochem. Eng. Asp. 2010, 367, 155-160. [CrossRef]

31. Zhou, X.; Li, H.; Xiao, H.; Li, L.; Zhao, Q.; Yang, T.; Zuo, J.; Huang, W. A Microporous Luminescent Europium Metal-Organic Framework for Nitro Explosive Sensing. Dalton Trans. 2013, 42, 5718-5723. [CrossRef] [PubMed]

32. Lu, Y.; Yan, B. A Ratiometric Fluorescent PH Sensor Based on Nanoscale Metal-Organic Frameworks (MOFs) Modified by Europium(Iii) Complexes. Chem. Commun. 2014, 50, 13323-13326. [CrossRef] [PubMed]

33. Matsushita, A.F.Y.; Tapia, M.J.; Pais, A.A.C.C.; Valente, A.J.M. Luminescent Properties of Lanthanoid-Poly(Sodium Acrylate) Composites: Insights on the Interaction Mechanism. Polymers 2020, 12, 1314. [CrossRef]

34. Zhou, X.-H.; Li, L.; Li, H.-H.; Li, A.; Yang, T.; Huang, W. A Flexible Eu(Iii)-Based Metal-Organic Framework: Turn-off Luminescent Sensor for the Detection of Fe(Iii) and Picric Acid. Dalton Trans. 2013, 42, 12403-12409. [CrossRef] [PubMed]

35. Ahmed, A.A.; Gypser, S.; Leinweber, P.; Freese, D.; Kühn, O. Infrared Spectroscopic Characterization of Phosphate Binding at the Goethite-Water Interface. Phys. Chem. Chem. Phys. 2019, 21, 4421-4434. [CrossRef]

36. Yang, J.; Dai, Y.; Zhu, X.; Wang, Z.; Li, Y.; Zhuang, Q.; Shi, J.; Gu, J. Metal-Organic Frameworks with Inherent Recognition Sites for Selective Phosphate Sensing through Their Coordination-Induced Fluorescence Enhancement Effect. J. Mater. Chem. A 2015, 3, 7445-7452. [CrossRef]

37. Yang, J.; Wang, Z.; Hu, K.; Li, Y.; Feng, J.; Shi, J.; Gu, J. Rapid and Specific Aqueous-Phase Detection of Nitroaromatic Explosives with Inherent Porphyrin Recognition Sites in Metal-Organic Frameworks. ACS Appl. Mater. Interfaces 2015, 7, 11956-11964. [CrossRef]

38. Wu, Z.; Yang, H.; Pan, S.; Liu, H.; Hu, X. Fluorescence-Scattering Dual-Signal Response of Carbon Dots@ZIF-90 for Phosphate Ratiometric Detection. ACS Sens. 2020, 5, 2211-2220. [CrossRef] [PubMed] 